\title{
Revisión Teórica y Metodológica de las Dificultades en Psicoterapia: Propuesta de un Modelo Ordenador ${ }^{1}$
}

\author{
Theoretical and methodological review of therapeutic difficulties: A systematizing model
}

\author{
Pablo Herrera Salinas \\ Pontificia Universidad Católica de Chile, Chile \\ Olga Fernández González \\ Universidad Santo Tomás, Chile \\ Mariane Krause Jacob \\ Pontificia Universidad Católica de Chile, Chile \\ Oriana Vilches Álvarez \\ Universidad de Valparaíso y Universidad de las Américas, Chile \\ Nelson Valdés Paula Dagnino \\ Pontificia Universidad Católica de Chile, Chile \\ (Rec: 8 de junio de 2009 - Acep: 3 de noviembre 2009)
}

\begin{abstract}
Resumen
El presente artículo tiene como objetivo presentar el resultado de una revisión teórica de los conceptos asociados a dificultades en psicoterapia desde distintas perspectivas y niveles de análisis, para luego proponer una estructura que permita sintetizar, diferenciar y comparar los distintos conceptos y hallazgos relevantes que tienen que ver con el amplio espectro de las dificultades en psicoterapia. Los conceptos relativos a las dificultades en psicoterapia fueron organizados como variables de input, proceso y output, dentro del proceso terapéutico, para finalmente realizar una discusión acerca de las implicancias teóricas, clínicas y especialmente metodológicas en el estudio de las dificultades en psicoterapia.

Palabras claves: Dificultades en psicoterapia, investigación en psicoterapia, metodología de investigación.
\end{abstract}

\begin{abstract}
This article presents a theoretical systematization of concepts regarding psychotherapeutic difficulties, from different perspectives and levels of analysis. It proposes a theoretical structure that allows comparing, differentiating and synthesizing the multiple concepts and empirical findings regarding psychotherapeutic difficulties. Concepts were organized as input, process and output variables along the therapeutic process, finally discussing theoretical, clinical and methodological implications in the study of therapeutic difficulties.

Key words: psychotherapy difficulties, psychotherapy research, research methodology
\end{abstract}

\footnotetext{
1 Trabajo realizado como parte del proyecto financiado por el Fondo de Ciencias y Tecnología, Chile, Fondecyt N ${ }^{\circ} 1080136$.

Correspondencia a: Pablo Herrera Salinas. E-mail: pabloherrera@vtr.net
} 


\section{Introducción}

\section{Polémicas en torno a las Dificultades en psicoterapia}

El estudio sistemático de las dificultades que inciden en el proceso terapéutico es reciente (Muran, 2002), siendo diversos los autores que han conceptualizado e investigado empíricamente el tema, desde distintas perspectivas teóricas y metodológicas. Esta heterogeneidad se ha traducido en múltiples conceptos con diversos grados de superposición y sin suficiente diferenciación metodológica y teórica (Bleuter, Moleiro, \& Talebi, 2002), pero a su vez han aportando riqueza al estudio del fenómeno.

En primer lugar, existen diferencias respecto de quién define, describe y califica las dificultades (paciente, terapeuta, observador externo), ya que para estos tres actores hay una relación distinta figura-fondo y se pueden hipotetizar diferentes distinciones e intereses (Herrera \& Araya, 2007; Muran, 2002). A su vez, hay diferencias respecto de dónde se ubica el foco de estudio, lo que a su vez se relaciona con la atribución del "origen" o "causa" de la dificultad. Así es como algunos investigadores se concentran en el paciente, con términos como: "resistencia", "reactancia", "refusal", "paciente difícil", etc. (Beutler et al., 2002), mientras otros han puesto el foco en el terapeuta, hablando de "contraactuación" o "errores técnicos" (Kottler \& Blau, 1989). Especialmente en los últimos años se ha puesto el énfasis en aspectos relacionales, acuñándose conceptos tales como "ruptura de la alianza", "impasse", "incomprensiones", “desencuentros", etc. ' (Safran \& Muran 2000).

También existen diferencias entre los investigadores en cuanto al marco temporal en que se observan las dificultades: antes de comenzar el proceso psicoterapéutico, durante el proceso y como resultado de éste. Otros autores se enfocan en micro-momentos difíciles (Stern, Sander, Nahum, Harrison, Lyons-Ruth, Morgan, Bruschweiler-Stern, \& Tronick, 1998); o buscan momentos e interacciones breves durante la sesión (Krause, Echávarri, Ramirez, Valdés \& Vilches, 2007) y también hay quienes observan la dificultad a lo largo del tratamiento, en fases compuestas de múltiples sesiones (Etchegoyen, 1986).

Por otro lado, se pueden distinguir numerosas posiciones teórico-clínicas respecto del significado que tienen las dificultades en el proceso psicoterapéutico (Arkowitz, 2002; Beutler et al., 2002; Kottler, 1992). Para algunos las dificultades en psicoterapia señalan un contenido reprimido importante (Freud, 1926), para otros, una "palanca de cambio"

\footnotetext{
En estas distintas miradas también se pueden distinguir posiciones epistemológicas implícitas, ya que mientras algunos plantean características "intrínsecas" en los actores como objetos, otros postulan una visión más constructivista o intersubjetiva y afirman que tanto el cambio como las dificultades surgen de los procesos interaccionales e intersubjetivos entre paciente y terapeuta (Duncan et al 1997; Stern et al 1998; Van Denburg \& Kiesler 2002).
}

(O'Hanlon, 1993), o una señal del paciente al terapeuta para que cambie el foco o la forma de sus intervenciones (Norcross, 2002). Incluso, desde una visión más radical, de Shazer (1984) propone abandonar los conceptos que se enfocan en procesos homeostáticos y/o feedback negativo y reemplazar la "resistencia" por la "cooperancia".

Ante esta enorme heterogeneidad teórica y metodológica para abordar el tema de las dificultades en psicoterapia, nos hemos planteado como objetivo de este artículo proponer una estructura que permita sintetizar, ordenar, diferenciar y comparar los distintos conceptos y hallazgos relevantes que tienen que ver con el amplio espectro de las dificultades en psicoterapia. En el próximo apartado se plantea la propuesta de ordenamiento.

\section{Propuesta de Ordenamiento}

Frente a la diversidad presentada, se propone una definición trans-teórica de "dificultades en psicoterapia", que dé cabida a la multiplicidad de miradas y conceptualizaciones, sin perder su aporte específico. Luego, se presenta una serie de criterios para diferenciar, ordenar y comparar los distintos conceptos relacionados.

Entonces, se entenderá por "dificultad en psicoterapia":

Todo fenómeno o factor que obstaculiza los mecanismos y procesos de acción y de cambio en psicoterapia, de acuerdo a la perspectiva de un observador particular y según la teoría implícita o explícita sobre cómo se produce el cambio. Asimismo, se considerará "dificultad en psicoterapia" un fenómeno o factor que se relaciona a resultados negativos del proceso o a una disminución en el avance o logros esperados.

Estas dificultades pueden expresarse clínicamente de múltiples maneras: (a) Estados displacenteros-disfóricos: tensión, ansiedad, desmotivación, aburrimiento, en uno o ambos actores; (b) Problemas relacionales: dificultad en la comunicación y entendimiento, dificultad en la negociación de las necesidades, amenazas al vínculo terapéutico; (c) Obstaculización del trabajo e intervenciones terapéuticas: evitación de trabajar, de pensar, de asociar, negación en la realización de tareas terapéuticas, rompimientos de los acuerdos o compromisos, etc; y (d) Ausencia de cambio cuando éste sería esperable (según un observador particular y desde su teoría del cambio).

Con el fin de sistematizar y comparar los múltiples conceptos revisados, se proponen como ejes ordenadores los siguientes criterios [ver Figura 1]:

1. Sistema o unidad de análisis donde se observa la dificultad: sistema individual, sistema terapéutico-relacional y sistema macro-social o contexto general.

2. Etapa del proceso de psicoterapia: input (aspectos del paciente, del terapeuta y/o del contexto, que existen independiente y previamente a la terapia), proceso 
Figura 1

Modelo Ordenador de las dificultades en psicoterapia.

\begin{tabular}{|c|c|c|c|c|}
\hline $\begin{array}{l}\text { Etapas } \\
\text { del } \\
\text { proceso }\end{array}$ & $\begin{array}{c}\text { Sistema / } \\
\text { Unidad Análisis }\end{array}$ & Concepto & Autor(es) & Nivel de Análisis Temporal \\
\hline \multirow{7}{*}{ 占 } & \multirow{4}{*}{ Paciente } & Pacientes Difíciles & Kottler (1992) & Historia de Vida; durante la terapia \\
\hline & & $\begin{array}{l}\text { Pacientes Visitantes - pacientes } \\
\text { demandantes }\end{array}$ & De Shazer (1988) & Momentos y fases de la terapia \\
\hline & & $\begin{array}{l}\text { Pacientes pre-contemplativos y } \\
\text { contemplativos }\end{array}$ & Prochaska (1983) & Momentos y fases de la terapia \\
\hline & & $\begin{array}{l}\text { Pacientes sin indicación de } \\
\text { psicoterapia }\end{array}$ & $\begin{array}{l}\text { Truant (1999) } \\
\text { Gans y Alonso (1998) }\end{array}$ & Historia de vida; fases de la terapia \\
\hline & \multirow{2}{*}{ Terapeuta } & Terapeuta menos efectivo & $\begin{array}{l}\text { Bleuter et al (2004) } \\
\text { Roth y Fonagy }(2005)\end{array}$ & $\begin{array}{l}\text { Durante la terapia; } \\
\text { outcome }\end{array}$ \\
\hline & & $\begin{array}{l}\text { Desajustes socioculturales con el } \\
\text { paciente }\end{array}$ & $\begin{array}{l}\text { Thoits (1985) } \\
\text { Beutler et al (1993) }\end{array}$ & Historia de vida; fases de la terapia \\
\hline & Contexto & $\begin{array}{l}\text { Cobertura y Previsión en Salud } \\
\text { Mental }\end{array}$ & $\begin{array}{l}\text { Thoits (1985) } \\
\text { Herrera y Araya (2007) }\end{array}$ & \\
\hline \multirow{15}{*}{ 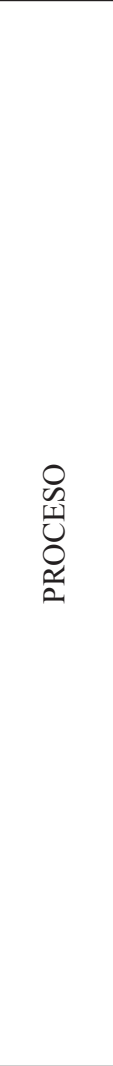 } & \multirow{8}{*}{ Paciente } & Resistencia & $\begin{array}{l}\text { Freud (1926) } \\
\text { Beutler et al (2002) } \\
\text { Arkowitz (2002) }\end{array}$ & $\begin{array}{l}\text { Momentos en sesión; } \\
\text { fases de la terapia }\end{array}$ \\
\hline & & Acting out & Etchegoyen (1986) & Momentos en sesión y entre sesiones \\
\hline & & Reacción terapéutica negativa & Etchegoyen (1986) & $\begin{array}{l}\text { Momentos en sesión; fases de la } \\
\text { terapia }\end{array}$ \\
\hline & & Reversión de la perspectiva & Etchegoyen (1986) & Fases de la terapia \\
\hline & & Reactancia & Brhem \& Brhem, 1981 & Momentos en sesión \\
\hline & & Refusal & Billow (2007) & Momentos en sesión \\
\hline & & Recaída & Prochaska (1983) & Entre sesiones \\
\hline & & Estancamiento & Krause et al (2007) & Micro momentos en sesión \\
\hline & \multirow{2}{*}{ Terapeuta } & Error Técnico & Kottler \& Blau (1989) & $\begin{array}{l}\text { Micro momentos; momentos en } \\
\text { sesión; fases de la terapia }\end{array}$ \\
\hline & & $\begin{array}{l}\text { Contratransferencia negativa y } \\
\text { Contraactuación }\end{array}$ & $\begin{array}{l}\text { Freud } \\
\text { Shoenewolf (2005) }\end{array}$ & $\begin{array}{l}\text { Micro momentos; momentos en } \\
\text { sesión; }\end{array}$ \\
\hline & \multirow{5}{*}{$\begin{array}{l}\text { Sistema } \\
\text { Relacional } \\
\text { Terapéutico }\end{array}$} & Impasse como falla en la sintonía & Stolorow y Atwood (1992) & $\begin{array}{l}\text { Micromomentos; } \\
\text { momentos en sesión }\end{array}$ \\
\hline & & Rupturas en la alianza & $\begin{array}{l}\text { Safran y Muran (2000); Muran } \\
(2002)\end{array}$ & $\begin{array}{l}\text { Micromomentos; } \\
\text { momentos en sesión }\end{array}$ \\
\hline & & Impasse como proceso sin avance & Etchegoyen (1986) & Fases de la terapia \\
\hline & & Desencuentros & Boston Change Group & Micromomentos \\
\hline & & Incomprensiones & Hill (2005) & Micromomentos; momentos en sesión \\
\hline \multirow{5}{*}{ 占 } & \multirow{3}{*}{ Paciente } & Fracaso Terapéutico & $\begin{array}{l}\text { Kottler \& Blau (1989); Strupp } \\
\text { (1975); Strupp \& Hadley } \\
\text { (1985); Deutch (1984) }\end{array}$ & Outcome \\
\hline & & Abandono Temprano(dropout) & (Bean 2005) & Fases de la terapia \\
\hline & & Resultado negativo (iatrogenia) & Franks \& Mays (1985) & Outcome \\
\hline & Terapeuta & Burnout & & Fases de la terapia \\
\hline & Contexto & [Ausencia de información] & & \\
\hline
\end{tabular}


(aspectos que ocurren en el proceso mismo: contrato, intervenciones terapéuticas, vínculo e interacciones), y output (aspectos que se presentan como resultados del proceso) (Orlinsky, Ronnestad, \& Willutzki, 2004).

3. Nivel de análisis temporal donde se presenta el fenómeno: procesos de micro-cambios, momentos en sesión, sesiones, fases o etapas de la terapia o durante todo el proceso (Elliott, 1991).

\section{Presentación y Revisión de Conceptos}

\section{Variables de "Input"}

Siguiendo la clasificación de Orlinsky et al. (2004), las variables de "input" son aquellas asociadas a características del paciente, a características del terapeuta, y a características del contexto institucional y macrosocial donde se realiza la intervención, que están presentes con anterioridad al inicio formal del proceso de psicoterapia.

\section{Sistema individual - paciente.}

¿Existen pacientes que por sus características son "en sî" difíciles para la mayoría de los psicoterapeutas? En general, cuando se consulta a los profesionales, éstos reportan la experiencia subjetiva de lidiar con "pacientes difíciles", más allá de posibles conflictos contra-transferenciales, errores técnicos o falta de indicación para el tratamiento (Bongar et al., 1991; Franks \& Mays 1985, Gold 1995; Munich \& Allen, 2003; Wong, 1983). Sin embargo, algunos cuestionan esta postura tildándola de positivista e ingenua, al ignorar la contribución del terapeuta y atribuir la dificultad a factores "intrínsecos" del paciente, contribuyendo a su estigmatización ${ }^{3}$ (Duncan, Hubble \& Miller, 1997; Najavits, 2001).

Más allá de la controversia epistemológica, tanto al realizar estudios de regresión estadística (Clarkin \& Levi, 2004) como al revisar la experiencia clínica acumulada (Kottler \& Carson, 2003), se puede observar que hay ciertas características de los pacientes que son presumiblemente previas al inicio de la psicoterapia y que se asocian al éxito y fracaso de la misma, dando cuenta del $30 \%$ de la varianza en los resultados (Asay \& Lambert, 1999).

Aunque la diversidad de estudios y focos es enorme (Clarkin \& Levi, 2004; Franks \& Mays, 1985; Kottler, 1992; Munich \& Allen, 2003), en las distintas investigaciones se puede concluir que los pacientes difíciles poseen al menos una de las siguientes características: (a) Síntomas graves y prolongados (Franks \& Mays, 1985; Wong, 1983); (b) Baja motivación para el cambio y la terapia, locus de control externo respecto de su problema, o dificultad para

\footnotetext{
Kottler (1992) hace una interesante revisión de los nombres -muchas veces denigratorios- que los terapeutas le hemos dado a los pacientes "difíciles": insoportable, odioso, rechazante de la ayuda, manipulador, imposible, "barracudas", renuente, resistente, desmotivado, etc.
}

la introspección y expresión (Gold, 1995; Kottler, 1992; Powell, 1995); (c) Dificultades generales en el establecimiento y mantención de relaciones interpersonales (Clarkin \& Levi, 2004; Franks \& Mays, 1985; Truant, 1999).

En función de la motivación al cambio del cliente, de Shazer (1988) propone los términos de cliente visitante (inicia la terapia sin percibir un problema ni considerarse parte de la solución); y cliente demandante (percibe un problema, pero no se considera parte de la solución). Esta conceptualización se complementa con las etapas de precontemplación o contemplación (Proshaska \& DiClemente,1983), que incluye a los clientes que llegan a terapia antes de percibir un problema o cuando no están decididos a cambiar. Este tipo de pacientes ha sido calificado como el más difícil para los psicoterapeutas novatos (Herrera \& Araya 2007).

Por otra parte, en términos diagnósticos, los pacientes que más han sido catalogados con el rótulo de "pacientes difíciles" han sido los diagnosticados con trastorno limítrofe de la personalidad, reportándose en ellos hasta un $66 \%$ de tasa de abandono en los tres primeros meses de tratamiento ${ }^{4}$ (Franks \& Mays 1985).

Finalmente, se distingue entre "paciente difícil" y " $p a$ ciente sin indicación para psicoterapia", en general o para un modelo de psicoterapia en particular, como es el caso de las psicoterapias breves y/o de tiempo limitado (Truant 1999; Clarkin \& Levi 2004).

\section{Sistema individual - terapeuta.}

Respecto de la importancia de las variables del terapeuta, se ha concluido que las características personales del terapeuta tienen más peso que el tipo de psicoterapia que practica. Algunos terapeutas individuales, independientemente de su enfoque teórico, producen resultados consistentemente mejores, mientras que otros producen efectos consistentemente negativos, especialmente en combinación con cierto tipo de pacientes (Beutler et al., 2004; Franks \& Mays, 1985; Najavits, 2001). Sin embargo, a diferencia de lo que ocurre con los pacientes, no se ha podido definir un perfil de "terapeuta difícil", y tal como afirman Roth y Fonagy (2005), en general los hallazgos no son concluyentes ${ }^{5}$.

De las variables estudiadas en los "terapeutas menos efectivos", se encuentra la experiencia y experticia del terapeuta. Estas variables parecen tener cierta influencia en la terapia, ya que los terapeutas más experimentados logran buenos resultados con un mayor porcentaje de pacientes, teniendo los pacientes de los novatos un éxito equivalente, pero un número mayor de abandonos al tratamiento (Roth \& Fonagy 2005).

\footnotetext{
${ }^{4}$ En contraste con el $14 \%$ de los diagnosticados con esquizofrenia y el $38 \%$ de los diagnosticados con otro trastorno de la personalidad.

5 En el sentido de que los estudios tienen pobre potencia estadística, deficiencias metodológicas y además hay resultados opuestos entre un estudio y otro.
} 
En relación a otras variables de la persona del terapeuta estudiadas, se ha visto que los terapeutas inefectivos tienden a tener menor grado de bienestar vital general (Beutler et al., 2004) y mayor presencia de rasgos como frialdad, pesimismo, inautenticidad, narcisismo y baja introspección (Strupp \& Hadley, 1985).

Finalmente, en relación a valores, actitudes y cultura, se ha visto que las diferencias demográficas y socioculturales entre paciente y terapeuta (des-ajuste sociocultural terapeuta/ paciente) afectan la capacidad de retención y motivación del paciente, asociándose con un peor pronóstico (Beutler et al., 1993; Franks \& Mays, 1985; Kottler, 1992).

\section{Sistema macro - social.}

La investigación en las dificultades relacionadas con el contexto donde se realiza la psicoterapia tiene ya más de dos décadas, y sus resultados indican dos grandes áreas de dificultades. Una tiene que ver con las deficiencias en las redes de apoyo del paciente (red social, familiar, socioeconómica y/o laboral), y la otra se relaciona con el sistema social de cobertura y previsión de la salud mental: políticas de atención, contexto de atención, derivación y coordinación de la red profesional (Franks \& Mays, 1985).

\section{Variables de Proceso}

La mayor parte de las conceptualizaciones e investigaciones empíricas sobre dificultades en terapia se centran en fenómenos y variables durante el proceso terapéutico, ya sea en sesión o durante un período o fase de la terapia. En este apartado, se analizarán los fenómenos estudiados tanto en un nivel de sistema individual (terapeuta y paciente por separado) como de sistema terapéutico completo (visión relacional).

\section{Sistema individual - paciente.}

Esta sección se centra en el estudio de las dificultades durante el proceso de terapia, con el foco puesto en el paciente. Se inicia con los conceptos que se han desarrollado para comprender las dificultades desde esta perspectiva.

El primer y más clásico concepto a revisar es el de resistencia, que aunque ha sido cuestionado especialmente desde fuera del psicoanálisis (de Shazer, 1984, Lazarus \& Fay, 1982), todavía sigue vigente (Beutler et al., 2002). Fue originalmente definido por Freud (1926) como la evitación inconsciente del trabajo analítico, por protección ante la angustia que genera la proximidad con el contenido reprimido. Desde una perspectiva supra-teórica se puede conceptualizar como señal general de ambivalencia o conflicto frente al cambio de parte del cliente (Arkowitz, 2002).

También desde una visión psicoanalítica, Etchegoyen (1986) define tres clásicos conceptos como "estrategias defensivas del yo", fenómenos atribuibles al paciente que tratan de impedir el desarrollo del insight. El acting out es un acto neurótico en el cual el pensamiento y la comunicación se reemplazan por la acción, afectando la tarea analítica. Por otro lado, la reacción terapéutica negativa se refiere a una respuesta paradójica o incoherente en que el paciente empeora o critica el tratamiento luego de mejorar, afectando los logros del análisis. Finalmente, la reversión de la perspectiva representa situaciones donde entre terapeuta y paciente hay un acuerdo manifiesto que oculta una discrepancia radical donde el paciente configura un contrato terapéutico paralelo e incompatible con el contrato analítico y los objetivos acordados con el terapeuta.

Otro concepto relevante es el de reactancia, definido como una reacción del sujeto ante la amenaza a la propia libertad, como una respuesta para recuperar la propia autonomía o algún aspecto importante de su identidad (Cowan \& Presbury, 2000), por lo que no tendría una connotación patológica desde una visión humanista-existencial.

La reactancia se debe diferenciar del concepto de refusal (traducible como "negativa" o "rechazo" (Billow, 2007), que se refiere a una deliberada no participación en descubrir o aprender acerca del material psíquico, con el objetivo de suspender o bloquear la actividad mental productiva, bajo el supuesto que hacerlo podría ser poco agradable o producir dolor, relacionado a un específico tema traumático. El refusal existe en el mundo conciente, pre-consciente e interpersonal, manifestándose de forma explícita (oposición, "yo no deseo hablar de esto") y/o encubierta (incumplimiento y retirada emocional, pseudo cumplimiento donde lo que se dice o siente más bien sirve de distractor u obstrucción).

Desde una perspectiva transteórica y procesual, Prochaska y DiClemente (1983) plantean una concepción de recaída como parte normal del tratamiento que, aunque implica una dificultad para el terapeuta, consta de múltiples vaivenes a lo largo de las cinco etapas del proceso de cambio que propone el autor. En este sentido, recaída sería un retroceso a una etapa anterior en el proceso de cambio.

Para finalizar con los conceptos desarrollados que se centran en las dificultades desde el paciente, presentamos el término estancamiento (Krause et al., 2007), que se refiere a la reiteración persistente del paciente, durante la sesión, de un patrón de funcionamiento (cognitivo, emocional o comportamental) relacionado con su problema e incompatible con el cambio. Este concepto comparte algunas características con el de impasse ${ }^{6}$ en el sentido que ambos se definen como un "no cambio". Sin embargo, el concepto de estancamiento se estudia en microprocesos en sesión ("episodios" de 3 minutos aprox.), no involucra al terapeuta y prescinde de una perspectiva teórica específica. El "impasse" de Etchegoyen (1986) se estudia a lo largo de fases prolongadas del proceso terapéutico $\mathrm{y}$, a pesar de que se observa mirando al paciente, explícitamente involucra a ambos actores.

\footnotetext{
Descrito en la sección de Sistema terapéutico - relacional.
} 


\section{Sistema individual - terapeuta.}

Esta sección se refiere al estudio de las dificultades en la terapia, situando el foco en el terapeuta, su conducta y experiencia durante el proceso de la terapia. En este contexto, la pregunta clave es: ¿cómo puede el terapeuta, en sesión, obstaculizar el progreso de la terapia?.

La investigación sobre los errores técnicos presenta algunas dificultades metodológicas, debido a que lo que se entiende por "error técnico" es completamente distinto según la orientación teórica de quien juzga el error ${ }^{7}$ (Kottler \& Blau, 1989; Kottler \& Carson, 2003). Algunas investigaciones se centran en la adherencia o distancia a un manual de tratamiento, mientras que otras se orientan al proceso interno de "la persona del terapeuta", que puedan redundar en errores durante el proceso terapéutico (Goldberg, 1990; Misch, 2000; Shoenewolf, 2005). Por ejemplo, la contraactuación o contra-resistencia, conceptos psicoanalíticos que se refieren al error del terapeuta de actuar los sentimientos e impulsos evocados en la situación con el paciente, repitiendo o confirmando los esquemas e identificaciones puestos en juego ya sea de parte del paciente o del mismo terapeuta (Reich, 1973; Schlesinger, 2003). En general se usa para describir situaciones donde el terapeuta inconscientemente comienza a jugar un rol en el mundo relacional del paciente, confirmando sus expectativas y esquemas.

Otra diferencia fundamental es la unidad de análisis de observación de los errores. Hay investigaciones que analizan las conductas específicas y técnicas concretas observables durante micro-etapas de la sesión (Alvear, 2005; Najavits \& Strupp, 1994); otras analizan estrategias terapéuticas distinguibles en unidades de análisis más prolongadas dentro del proceso, durante etapas o fases de la terapia (Kottler \& Blau, 1989; Kottler \& Carson, 2003); finalmente, se pueden distinguir otras investigaciones que se refieren a errores relacionados con creencias y actitudes del terapeuta subyacentes a su modo de hacer terapia (Ellis, 1985; Misch, 2000). Lamentablemente, la mayoría de las investigaciones que describen errores técnicos, mezcla estos niveles.

No obstante estas dificultades, es posible resumir los errores más frecuentes que cometen los terapeutas (Goldberg, 1990; Herrera \& Araya, 2007; Kottler \& Blau, 1989; Misch, 2000):

1. Interferencia de los propios conflictos no trabajados.

2. Expectativas irrealistas de éxito y competencia profesional instantánea. Creencia de que el éxito depende de la correcta aplicación de la técnica y procedimientos aprendidos.

\footnotetext{
7 En este sentido, es recomendable la revisión de la exhaustiva investigación transteórica de Strupp \& Hadley (en Franks \& Mays, 1985), quienes integran y ordenan los errores técnicos más frecuentes luego de consultar a gran cantidad de terapeutas de distintas orientaciones.
}

3. Exceso o falta de confianza en sus propias capacidades, asumiendo toda la responsabilidad por la "cura" del paciente.

4. Miedo a la Confrontación, dificultad en el manejo de la relación y del trabajo con el "aquí y ahora" de la sesión; errores de timing, impaciencia y "empujar" el proceso.

5. Errores de Diagnóstico: Buscar patología; sobrediagnosticar "resistencias" del paciente; confundir etiqueta con persona.

Por otro lado, se han descrito errores terapéuticos que no son tan frecuentes, pero son más graves, en el sentido que implican un riesgo de iatrogenia y generan deterioro en el paciente, como por ejemplo el usar técnicas orientadas a desafiar las defensas con pacientes que presentan patología más severa. Estos errores tienden a provocar efectos negativos transitorios o generan un abandono de la terapia, sin tener consecuencias permanentes excepto en el caso de los pacientes de alto riesgo, quienes sí pueden sufrir consecuencias permanentes o de largo alcance (Beutler et al, 1993; Franks \& Mays, 1985).

\section{Sistema terapéutico - relacional.}

En este apartado se presentan las dificultades del proceso de terapia como un fenómeno relacional, observando al sistema terapéutico como un todo y profundizando en diversos conceptos que se han desarrollado para comprender los quiebres en esta relación, principalmente por autores que se adscriben a la línea del psicoanálisis relacional o intersubjetivo.

Comenzaremos con el concepto de "impasse", que tiene un origen psicoanalítico y que se refiere específicamente a aspectos de la relación de paciente y terapeuta. Etcheghoyen (1986) define este concepto como algo distinto a la resistencia y al error técnico, por lo que no es asignable a ninguno de los actores por separado y se debe considerar como una variable propia del sistema terapéutico, aunque no aluda a ningún proceso explícito relacional y de hecho se estudie observando principalmente al paciente. Él lo define como una situación donde el trabajo analítico se realiza y sin embargo no hay avance, o donde había avance, y de pronto el proceso se detiene. Esta visión tiene implícito el que el estado normal de la terapia sea el avance gradual, y de hecho el autor afirma que se puede confundir con la marcha natural del análisis. Sin embargo, se diferencia en que no hay elaboración (es su reverso) y en que no hay progreso por un número prolongado de sesiones, repitiéndose las fases del tratamiento sin que se note un cambio a lo largo del tiempo ${ }^{8}$. No obstante este criterio temporal, el autor plantea que es muy difícil de diagnosticar y generalmente pasa desapercibido para el analista novel.

\footnotetext{
8 El autor ilustra el concepto con la metáfora de un hámster girando a gran velocidad la rueda de su jaula, sin moverse hacia ningún lado.
} 
Desde la mirada de los psicoanalistas intersubjetivos (Cowan \& Presbury 2000; Stolorow \& Atwood, 1992), el fenómeno del impasse se entiende como una falla en la sintonía entre paciente y terapeuta. Plantean que este impasse se manifiesta en la transferencia del paciente, y que es una respuesta a alguna actividad del terapeuta que el paciente experiencia como potencialmente retraumatizadora, por lo que éste toma las medidas para prevenir una fragmentación o aniquilación. Cuando paciente y terapeuta están "mal sintonizados", el miedo a ser re-traumatizado no es percibido por el terapeuta, y las dinámicas de protección y resistencia toman el primer plano en la relación, dejando ocultas las fallas en el proceso interaccional de desarrollo del self.

Otras investigaciones han explorado las dificultades en la relación desde la perspectiva de los pacientes (Muran, 2002; Petersen, 1998; Weiner, 1974). Algunas de las conclusiones más interesantes al respecto son: (a) los impasses son eventos altamente emocionales para los clientes, que si no se trabajan pueden llevar al abandono temprano de la terapia; (b) los pacientes distinguen cosas que los terapeutas hicieron o dijeron que les molestaron, sumado al reconocimiento de sus propias dificultades para expresar su insatisfacción y abrirse en terapia; (c) la mayoría de los pacientes piensa que los terapeutas no se dan cuenta del impasse; (d) en cuanto a las rupturas en la alianza, los pacientes tienden a reportar menos que los terapeutas.

Ahora, desde la mirada de la relación terapéutica como central en el proceso de cambio, uno de los conceptos más estudiados es la alianza terapéutica y las rupturas o "dysfluencies" en la alianza terapéutica (Muran, 2002; Safran \& Muran, 2000). Estos autores conceptualizan el estudio de las dificultades en psicoterapia como impasses y/o rupturas en la relación terapéutica, las que indican un quiebre o amenaza de quiebre en el proceso de negociación de las necesidades entre 2 individuos (paciente-terapeuta). Las rupturas reflejan formas de resolver la tensión dialéctica entre las necesidades de "agency" (autodirección, autodefinición o autogestión) y la necesidad de relacionarse, de entre los participantes. Existirían dos tipos característicos de rupturas: (a) retirada: cuando los pacientes se desenganchan de la terapia, de sus propias emociones o aspectos del proceso, privilegiando sus necesidades de relación por sobre las de "agency"; y (b) confrontación: cuando los pacientes expresan directamente angustia, resentimiento o insatisfacción con la terapia o con algunos aspectos de ella, favoreciendo sus necesidades de "agency" por sobre la relación.

Desde una exploración más microanalítica del proceso, el Boston Change Study Group ha aportado con el concepto de Desencuentros o encuentros fracasados (Stern et al., 1998). Ellos postulan que el cambio en psicoterapia se produce en momentos de encuentro privilegiados ("now moments"). Desde este contexto, se consideran como dificultades en psicoterapia los momentos en que se abre la posibilidad, pero el encuentro no se produce. Se describen estos momentos de no encuentro como: (a) Momentos perdidos: dejar pasar una oportunidad de encuentro, sin que éste ocurra; (b) Momentos fracasados: se reconoce la posibilidad de un momento de encuentro, pero no ocurre y se vuelve a vivir el patrón de desilusión o fracaso en la relación; (c) Momentos de encuentro señalados/nombrados, pero no producidos, porque la díada no está preparada; y (d) Momentos de encuentro en espera, cuando éste no se puede dar, no se puede compartir o no se puede resolver aún.

Otro aporte complementario desde una perspectiva de proceso lo provee Hill (2005) con su concepto de incomprensiones en el proceso terapéutico. La autora plantea que en la fase tres, llamada "Trabajo Terapéutico Central", uno de los componentes centrales es la superación de los obstáculos surgidos de las posibles incomprensiones en la relación paciente y terapeuta. En esta fase se observan niveles más altos de ansiedad que los observados anteriormente, y/o procesos de terapia detenidos.

\section{Variables de "Output" (Resultados)}

En este último apartado se agrupan los conceptos e investigaciones que estudian las dificultades y problemas en psicoterapia desde los resultados finales del proceso, con conceptos tales como fracaso terapéutico, abandono o deserción y resultado terapéutico negativo. Como se ha realizado hasta ahora, primero se presentarán los antecedentes con el foco en el paciente, luego en el terapeuta y finalmente en el contexto.

\section{Sistema individual - paciente.}

La investigación que estudia el resultado, comprensiblemente, se ha centrado casi exclusivamente en el impacto en el paciente, diferenciando el no éxito en distintas categorías que es importante distinguir. En primer lugar, se ha definido fracaso terapéutico como lo que ocurre cuando ambas partes involucradas concuerdan en que no se logró el cambio esperado, o no hubo un cambio positivo como producto de la terapia (Kottler \& Blau 1989). Aunque se pueden identificar múltiples indicadores de fracaso (Strupp \& Hadley, 1985), es finalmente el juicio del observador el que determina si corresponde catalogar así el resultado. Dado que en los ensayos clínicos entre el 40-70\% de los pacientes obtienen beneficios significativos, se puede esperar que más del 30\% de los pacientes obtengan resultados pobres o nulos (Lambert \& Ogles, 2004).

Por otro lado está el concepto de dropout o abandono temprano de la terapia, que hace referencia al porcentaje de pacientes que inician el tratamiento (o asisten a la entrevista de ingreso) y no lo terminan de común acuerdo con el terapeuta (Bean, 2005) .

\footnotetext{
9 Este concepto es útil para comprender las investigaciones sobre eficiencia y resultados terapéuticos, ya que algunas veces los datos se extraen solamente de los pacientes que completan el tratamiento ("completers"), mientras que otras veces se incluye como muestra la población que
} 
Por último, es importante distinguir entre resultado negativo e iatrogenia (Franks \& Mays 1985). El primer término hace referencia a cuando el paciente sufre un $d e$ terioro entre el inicio de la terapia y su finalización, pero no asigna causalidad, o sea, no culpa a la terapia por el deterioro. En cambio, el término "iatrogenia" implica que la terapia impactó negativamente en el paciente, causando su malestar. En general los estudios reportan que aproximadamente un 5-10\% de los pacientes empeoran luego del tratamiento (Lambert \& Ogles, 2004).

\section{Sistema individual - terapeuta.}

En lo que respecta al terapeuta, las investigaciones son inexistentes ${ }^{10}$, y en este sentido concordamos con la visión de Orlinsky (2006) de que esta es un área que requiere mayor exploración en la investigación de los resultados.

\section{Sistema macro - contexto.}

En el análisis de los resultados de la terapia en el contexto macro del paciente, sólo hay escasas investigaciones que exploran el efecto del tratamiento en el entorno familiar inmediato, especialmente en su cónyuge y en la relación de pareja (Franks \& Mays, 1985). Estos autores advierten este vacío y enfatizan la necesidad de evaluar el impacto de la terapia al menos en el sistema familiar nuclear del paciente identificado. Además, según Orlinsky (2006), es necesario investigar más sobre los posibles efectos de la terapia en el contexto más allá de la familia nuclear.

\section{Viñetas Clínicas}

A continuación se presenta una serie de viñetas clínicas, extraídas de la experiencia psicoterapéutica de los autores, con el objetivo de ilustrar algunos de los conceptos presentados.

\section{Viñeta 1}

Mariela tiene 41 años y consulta por hipocondría y depresión. Su terapeuta era un psicólogo en práctica en su consultorio. En un inicio, la terapia avanza rápidamente, los síntomas depresivos disminuyen, se logra interrumpir parte del patrón familiar de soluciones intentadas para "corregir" la hipocondría de la paciente, y la relación con el terapeuta muestra rasgos de idealización y erotización. La paciente encuentra un proyecto que la motiva y le da nuevas esperanzas sobre el futuro. Invita al terapeuta a la inauguración de su proyecto y lo nombra en un emotivo discurso. Algunas sesiones después, la paciente pide al terapeuta que escriba un justificativo para el colegio de

abandona tempranamente la terapia, la cual generalmente se acerca a la mitad de los pacientes iniciales (Lambert \& Ogles, 2004).

${ }_{10}$ Tal vez se podrían considerar investigaciones sobre burnout profesional en este acápite, sin embargo, creemos que se escapan al foco de la presente revisión. su hijo para que no haga educación física. El terapeuta evalúa la situación y le responde que eso no corresponde. Ante esto, la paciente responde furiosa, reclama traición del terapeuta y una actitud poco apoyadora. Abandona la sesión con un portazo, y no aparece por el consultorio por varias semanas. De vuelta se logra recontinuar el trabajo. No obstante, un par de sesiones después del retorno, el terapeuta comienza a explorar la relación idílica que reporta la paciente con su madre. Ante la exploración, la paciente revela sórdidos y violentos detalles, revelando abusos físicos y psicológicos graves de parte de su madre. El terapeuta, sorprendido, confronta la contradicción en el discurso de la paciente. Ésta parece perturbada al retirarse de la sesión. Nunca más vuelve.

\section{Análisis}

Si analizamos las variables de input al proceso terapéutico, desde el punto de vista del paciente, tenemos que podemos considerar a esta paciente como difícil, en cuanto que presenta características de paciente borderline (idealización y devaluación), y como paciente demandante, en cuanto que percibe problemas (ej: sus "enfermedades") pero no su parte en la solución de los mismos. Desde el punto de vista del input del terapeuta, éste tiene poca experiencia en el manejo de pacientes con estas características. En cuanto a un análisis del proceso terapéutico, se podría hablar de acting out, en cuanto a su vivencia de la frustración. Desde el punto de vista de los sistemas relacionales en el proceso, tenemos una ruptura de la alianza, debido al error del terapeuta de confrontar directamente a la paciente frente al tema de su madre cuando ella aún no estaba preparada para tolerarlo, donde la paciente vivencia que sus necesidades no son consideradas por el terapeuta, fracasando la negociación. En la fase de output, se observa el fenómeno de abandono temprano de la terapia. Además el terapeuta queda afectado por la situación, con muchas ansiedades y ambivalencias acerca de su proceder.

\section{Viñeta 2}

Jorge es un psicólogo que consulta "porque todos los psicólogos debemos hacernos terapia". Es puntual, respetuoso y entrega mucho material para trabajar, evidenciando su capacidad de introspección y auto-reflexión, incluso soliendo frecuentemente auto-interpretarse y sugerirse tareas o ejercicios para su propio desarrollo. La terapeuta al comienzo se siente encantada de tener un paciente articulado y reflexivo, sin embargo a medida que avanzan las sesiones empieza a sentirse excluida del diálogo (como escuchando un monólogo), prescindible y frustrada por no poder aportar al proceso de Jorge. Cuando la terapeuta comunica algo que Jorge no ha pensado aún, se frustra ante la fría recepción de sus aportes, sintiéndose rechazada. Asimismo, cuando ésta reflexiona sobre el proceso terapéutico, siente que ha habido trabajo, elaboración y reflexión, pero no observa un 
cambio real, y tampoco una conexión emocional sentida de parte de Jorge.

\section{Análisis}

Desde el punto de vista del input, a primera vista no parece ser un "paciente difícil", aunque uno podría decir que no hay una motivación o necesidad realmente sentida de necesitar ayuda (paciente pre-contemplativo); o que se trata de un paciente con características narcisísticas, que obstaculizan el vínculo con otro. Desde el proceso terapéutico como un sistema relacional, se puede visualizar una ruptura de la alianza evitativa, ya que han prevalecido las necesidades del paciente; no hay confrontación directa, pero sí retirada afectiva. Finalmente, podría también conceptualizarse como impasse en el proceso, en el sentido de que hay movimiento, que no se observan cambios, y estancamiento, ya que el paciente persiste en sesión en su patrón problema de desconexión afectiva, sobre-intelectualización y estilo de relación centrado sólo en sí mismo.

\section{Viñeta 3}

Rodrigo, 17 años, su madre lo trae a consultar porque el joven está muy desmotivado, no quiere ir al colegio, no se levanta y está muy callado. La madre lo asocia a que el padre está en casa con una enfermedad terminal, a punto de morirse. Ella cree que necesita psicoterapia. El joven asiste puntualmente a las sesiones convenidas, incluso llega un poco adelantado. En sesión no habla nada, no contesta las preguntas, sólo accediendo a realizar algunos dibujos; durante las siguientes sesiones sólo dibuja, en silencio. Termina su proceso de 12 sesiones sin hablar.

\section{Análisis}

Se podría entender este proceso, desde el input del paciente, como un problema de motivación por consultar, o como ausencia de indicación para psicoterapia en este momento. Desde el punto de vista de proceso, con el foco en la persona del terapeuta, se podría entender como dificultad en la técnica de éste, en cuando no ha logrado construir la confianza y seguridad mínima en el vínculo como para que el joven confíe en hablar, pese a que el paciente acude regularmente. Tampoco el terapeuta consideró la posibilidad de derivarlo a otro terapeuta o psiquiatra, o trabajarlo desde una perspectiva familiar. Con la mirada en el proceso, pero con el foco en el paciente, se podría hablar de reactancia, porque se percibe la terapia como una "intromisión" en su espacio de autoconfiguración. También puede ser clasificado como un "refusal" u oposición deliberada a no participar en este proceso. Desde el punto de vista del proceso como sistema relacional, se podría entender como un fenómeno de estancamiento, ya que se mantiene isomórficamente la expresión del conflicto, no hay reconocimiento del problema ni nuevas reconceptualizaciones de éste, y se mantiene durante todo el tiempo que dura el trabajo pactado. Además, se podría entender como una falla en la sintonía paciente-terapeuta, no pudiendo el paciente romper el "miedo" a una experiencia de retraumatización de formas relacionales. Desde el output del proceso, se podría conceptualizar como un fracaso en los resultados, ya que la madre no refiere cambios importantes, sino más bien una mantención de sus conductas.

\section{Discusión}

Iniciamos la discusión con una pregunta que nos parece central ¿Es posible prescindir de una teoría del cambio y de la terapia para identificar y estudiar empíricamente las dificultades en psicoterapia? Esto se plantea considerando que lo que se entiende en la literatura por "dificultad en terapia" esencialmente se basa en dos criterios: (a) variable cuya presencia se relaciona negativamente con el éxito de la terapia, y (b) fenómeno que se percibe como obstaculizando los procesos de cambio y los mecanismos de acción de la terapia. Si se utiliza el primer criterio puramente estadístico, tal vez se puede prescindir de una teoría pero probablemente el valor clínico del estudio sea reducido. Por otro lado, si la conceptualización se basa en el segundo criterio, claramente se requiere tener y explicitar: (a) una teoría del cambio psicoterapéutico, y (b) una teoría de los mecanismos de acción de la terapia. Para ilustrar este punto, podemos pensar en un ejemplo simple (y algo exagerado): para un terapeuta altamente racional y que enfatiza la modificación cognitiva, es un "paciente difícil" quien está gran parte de la sesión llorando; en cambio, para un terapeuta experiencial ese paciente estaría "contactado", siendo un "paciente difícil" otro que se pase gran parte de la sesión en argumentos a nivel intelectual. Por otro lado, una mejoría rápida podría ser interpretada psicoanalíticamente como un impasse vía "fuga hacia la salud", mientras que sería un signo de éxito para un terapeuta estratégico. Es a partir de estos argumentos que se plantea la necesidad de explicitar una teoría de la terapia y del cambio terapéutico ${ }^{11}$ para futuros estudios, especialmente si se centran en el proceso psicoterapéutico y no exclusivamente en establecer variables predictoras del resultado terapéutico.

Además de la coherencia teórica entre concepción del cambio y dificultades en psicoterapia, se requiere una coherencia entre la definición del fenómeno a analizar y la metodología utilizada. Por ejemplo, si se realiza un microanálisis, sería imposible estudiar el concepto de Etchegoyen (1986) de "impasse", ya que éste por definición sólo se puede apreciar durante períodos largos y fases de la terapia, o bien, si el concepto es intrínsicamente relacional, como el de "rupturas de la alianza", no se debería observar por separado al paciente y terapeuta, sino en la interacción,

\footnotetext{
11 Esta teoría perfectamente puede ser transteórica, de factores comunes o inespecíficos, siempre cuando explicite lo que entiende por proceso terapéutico y qué es lo que ayuda y no ayuda al cambio.
} 
porque sino la metodología empleada distancia del fenómeno definido a observar.

Otro aspecto esencial a considerar en el diseño de las investigaciones en esta temática es la relevancia crítica del manejo que el terapeuta haga de ellas, y por lo tanto su resolución. Este aspecto, de obvias repercusiones clínicas, también tiene implicancias metodológicas, ya que sería poco productivo buscar una correlación directa simple entre situaciones difíciles y éxito terapéutico, pudiendo ser mucho más provechoso considerar la resolución o manejo de esas dificultades en el análisis, como un aporte a la clínica y a la formación de los terapeutas, como lo plantea Muran (2002).

Por otro lado, a nivel de temas de investigación, existe acuerdo con lo postulado por Orlinsky (2006) en el sentido de que hay una carencia en el estudio de las dificultades a nivel de resultados, ya que éstos se limitan casi exclusivamente al análisis del impacto de la terapia en los síntomas y problemáticas presentes del paciente. Por lo tanto, futuras investigaciones podrían ampliar el foco hacia los efectos en aspectos más amplios de la vida del paciente, así como efectos en el terapeuta y en el contexto micro y macro.

Finalmente, con relación a la utilidad e implicancias del uso de estos conceptos para la psicoterapia, se pueden establecer ciertas distinciones y precauciones. Por un lado, las tipologías sobre "pacientes difíciles" podrían ser de utilidad para seleccionar los casos que atienden los terapeutas en formación, así como para tener precaución sobre ciertas herramientas técnicas que pueden ser contraindicadas para casos graves. Sin embargo, más allá de este uso podemos concordar con Duncan et al. (1997) en que la misma existencia del concepto puede predisponer al psicoterapeuta a caer en sesgo confirmatorio y la profecía autocumplida con el paciente, desarrollando una contra-transferencia negativa, disminuyendo su motivación y esperanza en el cambio, afectando el vínculo, etc.

Por otro lado, en conceptos como el "desencuentro" y la "falla en la sintonía", se puede apreciar el aporte clínico de un microanálisis, al ayudar al psicoterapeuta a visibilizar esos momentos privilegiados en que se puede facilitar una experiencia emocional correctiva. Finalmente, luego de revisar distintas conceptualizaciones y diversos niveles de análisis y estudio de esta gran heterogeneidad de fenómenos, es necesario destacar que las dificultades, problemas, errores y fracasos en psicoterapia existen y deben ser visibilizados ${ }^{12}$ para poder estudiarlos y aprender de ellos. En este sentido, el reconocer las dificultades no sería simplemente "enfocarse en lo que no funciona" o "ver el vaso medio vacío", sino que es un aviso de algo importante. De esta forma, las dificultades se pueden conceptualizar

\footnotetext{
12 Esto, que puede parecer muy simple, no siempre se da, ya que por ejemplo, como observó Barbrack (1985), existe un sesgo negativo de publicación en nuestras revistas científicas cuando los resultados de las investigaciones no son exitosos.
}

como señales en el camino, oportunidades de profundizar e incluso facilitar una experiencia emocional correctiva tanto del paciente como del terapeuta.

\section{Referencias}

Alvear, K. (2005). Señalamientos a terapeutas principiantes sobre la dirección de la cura. Tesis (Psicología). Universidad de Santiago de Chile, Santiago, Chile.

Arkowitz, H. (2002). Toward an integrative perspective on resistance to change. Psychotherapy in Practice, 58, 219-227.

Asay, T. \& Lambert, M.(1999). The empirical case for the common factors in therapy: quantitative findings. En: Hubble, M., Duncan, B. \& Miller, S. (Eds). The Heart and Soul of Change. What Works in Therapy. Washington: American Psychological Association.

Barbrack, C. (1985). Negative outcome in behavior therapy. En: Mays, D. \& Franks C. (Eds.) . Negative outcome in psychotherapy and what to do about it (76-105). New York: Springer.

Bean, M.C. (2005). Choosing not to return: diverse student's intake experiences at a university counseling center. Dissertation (PhD), Ohio State University, Ohio, EEUU, 147.

Beutler, L., Malik, M., Alimohamed, S., Harwood, T., Talebi, H., Noble, S. \& Wong, E. (2004) Therapist variables. En: M. Lambert (Ed.). Bergin and Garfield's Handbook of Psychotherapy and Behavior Change. New York: Wiley and Sons.

Beutler, L, Machado, P. \& Allstetter, S. (1993) . Therapist Variables. En: Bergin, A. \& Garfield, S. (Eds.) Handbook of Psychotherapy and Behavior Change (4th Edition). New York: Wiley.

Beutler, L., Moleiro, C. \& Talebi, H. (2002) Resistance in Psychoterapy: What Conclusions are Supported by Research. Journal of Clinical Psychology, 58, 207-217.

Clarkin, J. \& Levi, K. (2004). The influence of client variables on psychotherapy. En Lambert, M. (Ed.). Bergin and Garfield's Handbook of Psychotherapy and Behavior Change. New York: Wiley and Sons.

Cowan, E. \& Presbury, J. (2000). Meeting Client resistance and Reactance with Reverence. Journal of Counseling \& Development, 78, 411-419.

de Shazer, S. (1984). The death of resistance. Family Process, 23, 92103.

de Shazer, S. (1988). Clues. Investigating solutions in brief therapy. New York: Norton.

Duncan B., Hubble M. \& Miller S. (1997) . Psychotherapy with “impossible cases". New York: Norton.

Elliot, J. (1991). Five dimensions of therapy process. Psychotherapy Research, 1(2), 92-103.

Ellis, A. (1985). Overcoming resistance. New York: Springer.

Etchegoyen, H. (1986). Los fundamentos de la técnica psicoanalítica. Buenos Aires: Amorrortu.

Franks, C. \& Mays, D. (Eds. 1985). Negative outcome in psychotherapy and what to do about it. New York: Springer.

Freud, S. (1926, 2001). Inhibición, síntoma y angustia. En: Obras Completas. Buenos Aires: Amorrortu.

Gold, J. (1995) Knowing and not knowing: commentary on the roots of psychotherapeutic failure. Journal of Psychotherapy Integration, 5(2), 167-170.

Goldberg, C. (1990). Typical mistakes of the seasoned therapist. En: Margenau, E. (Ed.) The Encyclopedic Handbook of Private Practice (785-798). New York: Gardner Press.

Herrera, P. \& Araya, C. (2007). Errores comunes de terapeutas novatos. Tesis (Magíster en Psicología Clínica). Universidad Adolfo Ibáñez, Santiago, Chile.

Hill, C. (2005). Therapist techniques, Client involvement, and the therapeutic relationship: inextricably intertwined in the therapy process. Psychotherapy. 42(4), 431-442.

Kottler, J. \& Blau D. (1989). The imperfect therapist. San Francisco: Jossey-Bass Publishers. 
Kottler, J. \& Carson, J. (2003). Bad therapy. Master therapists share their worst failures. New York: Brunner-Routledge.

Kottler, J. (1992). Compassionate therapy: working with difficult clients. San Francisco: Jossey-Bass Publishers.

Krause, M., Echávarri, O., Ramírez, I., Valdés, N. \& Vilches, O. (2007) Aprender a observar para hacer visible: Cambio y estancamiento en la terapia. En: $8^{\circ}$ Congreso Chileno de Psicoterapia: Convergencias y singularidades en psicoterapia (Viña del Mar, Chile). Trabajo no publicado.

Lambert, M. \& Ogles, B. (2004) The efficacy and effectiveness of psychotherapy. En Lambert, M. (Ed.) .Bergin and Garfield's Handbook of Psychotherapy and Behavior Change. New York: Wiley and Sons.

Misch, D. (2000). Great expectations: Mistaken beliefs of psychodynamic psychotherapists. American Journal of Psychotherapy, 54(2), 172-203.

Muran, J. (2002) A Relational Approach to understanding change: plurality and contextualism in a psychotherapy research program. Psychotherapy Research, 12 (2) 115-138.

Najavits, L. (2001) .Helping Difficult Patients. Psychotherapy Research, 11(2) pp. 131-152.

O’Hanlon, W.H. (1993) Raíces profundas. Principios básicos de la terapia y de la hipnosis de Milton Erickson. Barcelona: Paidós.

Orlinsky, D. (2006). Comments on the state of psychotherapy research (as I see it). Psychotherapy Bulletin, 41, 37-41.

Orlinsky, D., Ronnestad, M. \& Willutzki, U. (2004). Fifty years of psychotherapy process-outcome research: continuity and change. En: Lambert, M. (Ed.). Bergin and Garfield's handbook of psychotherapy and behavior change. New York: Wiley and Sons.
Petersen, D. (1998). Clients' perspectives on unresolved therapeutic impasses. Tesis (PhD). EEUU, University of Maryland College Park, 194

Powell, D. (1995) Lessons learned from therapeutic failure. Journal of Psychotherapy Integration, 5(2), 175-182.

Prochaska, J. \& DiClemente, C (1983) Stages and processes of self-change of smoking: Toward an integrative model of change. Journal of Consulting and Clinical Psychology, 51, 390-395.

Reich, A. (1973). Empathy and Countertransference. En: Psychoanalytic Contributions. New York: International University Press. 324-360

Safran, J. \& Muran, J. (2000) Negotiating the Therapeutic Alliance. A Relational Treatment Guide. New York: The Guilford Press.

Schlesinger, H. (2003). The Texture of Treatment: On the Matter of Psychoanalytic Technique. New Jersey: The Analytic Press.

Stern, D., Sander, L., Nahum, J., Harrison, A., Lyons-Ruth, K., Morgan, A., Bruschweiler-Stern, N. \& Tronick, E. (1998) Non-Interpretive mechanisms in Psychoanalytic Therapy. The something more than interpretation. International Journal of Psycho-Analysis. 79, 903-921.

Stolorow, R. \& Atwood, G. (1992) Los contextos del ser. Las bases intersubjetivas de la vida psíquica. Barcelona: Herder.

Strupp, H. \& Hadley, S. (1985) Negative effects and their determinants. En Mays D. \& Truant, G.S. (1999). Assessment of suitability for psychotherapy. American Journal of Psychotherapy, 53(1), 17.

Weiner, M.F. (1974). The psychotherapeutic impasse. Dis Nervous System, 35(6), 258-61.

Wong N. (1983). Perspectives of the difficult patient. Bulletin of the Menninger Clinic, 47(2), 99-106. 
